\title{
Nature, Our Climate and Our Health Risks
}

(BIRDEM Med J 2017; 7(3): 179-186)

A natural disaster is defined as an event of nature, which overwhelms local resources and threatens the function and safety of the community. Some diseases depend on suitable environment for their outbreak. We have 8 diseases those emerged in last few years. Our main natural disasters are related to flood and torrential rain and related events. Earth quake even tsunamis happened here but so far not to the devastating state. Technological disaster like collapse of big buildings unnoticed firebreak out in huge industry has claimed lives here and risked prevailing health system.

Deaths associated with natural disasters, particularly rapid-onset disasters particularly torrential rain, flood, cyclones are overwhelmingly due to blunt trauma, crush-related injuries, or drowning. The risk for outbreaks is often presumed to be very high in the chaos following natural disasters, a fear of misconception of association between dead bodies and epidemics. The availability of safe water and sanitation facilities, the degree of crowding and the availability of healthcare services all interact within the context of the local disease ecology to influence the risk for communicable diseases and death in natural disasters

Meliodosis is a prevailing condition but only recently has been well recognized by our clinicians. Viral infections like Japanese encephalitis, Nipah, Bird flu, Swine flu could raise concern among clinicians and public health department but not to the extent like Dengue and Chikungunya.

An alert and versatile public health approach to disaster risk management and mangement of climate and environment prone diseases could help the vulnerable communities in disaster and outbreaks of diseases

\section{Introduction}

- Natural disasters where the trigger is a natural phenomenon or hazard (biological, geological, or climatic) viz-cyclone;

- Technological disasters such as collapse of large building, chemical spills; or

- Complex emergencies such as civil wars and conflicts.

\section{Natural disasters}

a) Hydro-meteorological disasters like floods, are the most common (40 percent) natural disasters of Bangladesh and worldwide.

b) Geophysical disasters are the second-most reported type of natural disaster and earthquakes are the majority of disasters in this category. Tsunamis are commonly associated with earthquakes, they have a similar clinical and threat profile to that of tropical cyclones (e.g., typhoon or hurricane).

c) Geomorphologic disasters such as avalanches and landslides

All are associated with infectious disease transmissions and outbreaks but documentation is generally lacking.

Deadly (deadliest ever!) mudslide triggered by torrential monsoon rain in southeastern Bangladesh in recent days (June 2017 and later) have claimed many lives. It happened just two weeks after the Cyclone Mora (May 2017) (previously Cyclone Bhola 1970, Gorky 1991, Sidr 2007, Aila 2009, Roanu 2016). Renewed mudslides, collapsing hillsides and heavy flooding estimated to have cause further damage (UN RC Jun 2017).

Bangladesh experiences different types of natural disasters almost every year because of global warming and change of climate. Almost $80 \%$ of total area of the country is prone to floods/flash flood. South and southeastern part of the country were hit by cyclone and storm surges during last few years. Whole coastal belt along the Bay of Bengal is experiencing salinity intrusion. Whereas north and north western part of the country are experiencing extreme temperature and drought almost every year. Our climate supporting this year the outbreak of the vector born viral disease Chikungunya like it did for Dengue in the year 1999.

\section{Dead bodies and diseases}

The sudden presence of large numbers of dead bodies in the disaster-affected area may heighten concerns of 
disease outbreaks ${ }^{1}$ despite the absence of evidence that dead bodies pose a risk for epidemics after natural disasters. Dead bodies only pose health risks in a few situations that require specific precautions such as deaths from cholera or hemorrhagic fevers

\section{The immediate(direct) effects of natural disasters ${ }^{2}$}

Every disaster scenario is unique in its own way. As a basis for planning, it is nevertheless useful to consider the types of injuries the majority of the victims will incur in order to evaluate the immediate needs of the disaster response team.

\section{Floods/Climate disasters}

Include seasonal floods, hurricanes, and typhoons. Seasonal floods cause increased incidence of

diarrheal diseases, respiratory infections, dermatitis and snake bites. The risk of compromised water supplies depends on the condition of the community's water supply before the disaster.

The greatest risk of mortality from a flood is predictably drowning. In our place floods account for more yearly deaths than all other natural disasters. It takes less than two feet of moving water to float a large vehicle such as a school bus and only between three and six inches of fast moving water to sweep a large man off his feet.

Second only to drowning, exposure to different elements accounts for a large percentage of the deaths and critical injuries that occur in flood.

The public health consequences of flooding are disease outbreaks mostly resulting from the displacement of people into overcrowded camps and crosscontamination of water sources with fecal material and toxic chemicals. Flooding also is usually followed by the proliferation of mosquitoes, resulting in an up surgence of mosquito-borne diseases such as malaria.

\section{Hurricanes, Typhoons and Cyclones}

In a hurricane (cyclones in the Indian Ocean and typhoons in the western Pacific Ocean), the greatest mortality originates from the secondary disasters that have been triggered such as small tornadoes, flash flooding and storm surges. In coastal regions (within 30 miles of a salt-water coast), the level of a hurricane's storm surge is one of the strongest predictors of mortality. Historically, nine out of ten deaths form a hurricane can be directly accounted to the preceding storm surge. This effect can be exacerbated by superimposing wind waves upon the storm tidal height and the strong winds. Crush injuries are common in severe storms.

In a hurricane, many additional trauma incidents are caused by large objects which can become airborne in the severe winds. The most common non- fatal traumatic injury in a hurricane is superficial lacerations from flying glass and other debris. ${ }^{3}$

\section{Tornadoes}

Due to the lack of warning, a tornado offers a community little time to prepare or seek shelter and hence the morbidity and mortality is proportionally higher compared to other disasters. In the United States, the leading cause of death and critical permanent injury in a tornado is cranio-cerebral injury due to projectiles accelerated by tornado winds. Crush injuries due to collapsing structures or extremely large airborne debris are also frequent.

Lacerations, contusions, abrasions, punctures, and musculoskeletal strain account for over half of the total injuries seen in local emergency rooms. Most victims suffer from multiple wounds and many of the soft tissue injuries occur in areas of exposed skin such as the head and neck.

Tornado victims also exhibit a characteristic abrasion pattern on exposed skin that is due to fine particles of soil, mud, sand and even water which strike the body at highly accelerated speeds.

Wound contamination and subsequent infection, appear to be a major contributor to post operative sepsis leading to an increased need of aggressive wound care and surgical debridement.

One fifth of fracture injuries in the aftermath of a tornado can be expected to be open, contributing to the infection rates among patients.

\section{Mudslides/avalanches/landslides}

Mudslides, which are commonly seen in flooding situations as well, can lead to crush injuries, severe internal bleeding, multiple organ dysfunction syndrome, and asphyxiation. In many instances mudslides occur in conjunction with volcanic eruptions as the topographical contour of the hillside is changed rapidly. In our country this change has been speculated to be manmade. 


\section{Earthquakes/Collapse of large building}

Earthquakes induce a high level of mortality due to crush injuries from falling objects. If an earthquake occurs at night, there is a greater incidence of trauma to the pelvis, thorax and spine. These injuries commonly lead to severe damage to internal organs coinciding with severe internal bleeding. If the earthquake occurs during the daytime, it is more common to see injuries of the extremities with comorbid lacerations, severe external bleeding and crush injuries including rhabdomyolysis and compartment syndrome. Injuries during a diurnal earthquake occur to the skull and the collarbone because of falling debris. Victims that have been trapped in fallen rubble for hours or possibly days run the risk of having infected wounds and/or gangrene. Common trauma procedures that need to be urgently addressed include amputations, vascular stabilization, faciotomies, orthopedic stabilization and debridement and/or dressing of severe open wounds. It is important to note that patients who suffer from crush injuries also run a high risk of developing hypovolemic shock, hyperkalemia, renal failure and have a greater chance of having a fatal cardiac arrhythmia or myocardial infarct. ${ }^{3}$ Fire is another major concern within the first 'golden' 24 hours after these disasters, burn injuries, as well as respiratory problems due to smoke inhalation can quickly become a major strain on the medical system after an earthquake or sudden collapse of a large building. Outbreaks of infectious diseases may be reported when earthquake disasters result in substantial population displacement into unplanned and overcrowded shelters, the destruction of water/sanitation systems and the degradation of sanitary conditions directly caused by the earthquake.

Tsunamis are catastrophic tidal waves caused by earthquakes on the ocean floor. Waves can travel several hundred kilometers per hour and can be as much as 10 meters high when they reach shore. Tsunamis are commonly associated with earthquakes, they have a similar clinical and threat profile (water-related consequences) to that of tropical cyclones (e.g., typhoon or hurricane). Damage on shore can be extensive and usually the number of deaths far exceeds the number of survivors with severe injuries.

\section{Droughts}

In a region that has been severely affected by a drought, mortality may increase sharply due to protein malnutrition (kwashiorkor) or calorie malnutrition (marasmus). Certain vitamin deficiencies, such as a lack of vitamin A, can lead to specific consequences such as xerpohthalmia and child blindness. Long standing malnutrition can easily lead to increased rates of disease and mortality and limit the general population's functionality.

\section{Fire}

Bush fire or wild fire is not common in our place. However, large-scale urban fires are still possible so also those secondary to other natural disasters like earthquake and those of technological disaster like industrial fire and fire after a collapse of a large building with huge dwellers/workers. Injuries in a large-scale urban fire are more commonly burn injuries and deaths associated with asphyxiation. Common complications of burn injuries include hypovolemic shock as well as infection and sepsis.

\section{Immediate (indirect) medial consequences of natural disasters. $^{2}$}

a) Cardiac events: In the aftermath of a major natural disaster such as an earthquake, an increase in acute myocardial infarctions (AMI) is not uncommon. ${ }^{4}$ In the Taiwan Chi-Chi Earthquake of 1999, the sudden increase of heart attacks were limited to mostly male patients and was attributed mainly to formerly sedentary individuals becoming suddenly and intensely active throughout the rescue period. It has also been suggested that the physiological 'fight or flight' response that is initiated during times of perceived personal danger such as an earthquake or other natural disaster, is itself contributory to the increase of post catastrophe AMIs. The fight or flight response, which is a hallmark of systemic sympathetic nervous system activation, leads to an increased vulnerability to myocardial attacks in individuals with pre-existing heart disease. Along with outright heart attacks, other cardiovascular complications are increasingly seen after natural disasters such as unstable angina and potentially fatal arrhythmias.

b) Others: In many instances, the effect of the increased stress load is compounded with the interruption of regular medical services for pre-existing conditions such as diabetes mellitus and high cholesterol. 
In one study of rheumatoid arthritis (RA) patients showed that after an earthquake the incidence of RA activity (pain, stiffness and swelling) increased dramatically.

\section{Respiratory problems}

In some instances, respiratory conditions such as allergies and asthma are aggravated after a natural disaster. This is especially true if there has been largescale ejection of pollutants into the atmosphere as with a volcanic eruption or forest fire and industrial fire. In many studies, there was little to no appearance of $d e$ novo respiratory conditions among the general population who received minimal to medium amount of exposure to the airborne irritants. Respiratory illnesses start to show a substantial increase in the days to weeks after a disaster. This apparent shift in illness is attributed more to the transmission of communicable diseases such as influenza or even tuberculosis in crowded temporary shelters. Likewise, respiratory illnesses have been noted to particularly increase in the extended aftermath of a flood. As the flood water recedes and victims reclaim their former living areas, the concentration of allergens such as dust mites climb dramatically within washed out homes, schools and offices.

\section{Infections}

The risk factors for increased infectious diseases transmission and outbreaks are mainly associated with the after-effects of the disasters rather than to the primary disaster itself or to the corpses of those killed.

\section{Diarrheal diseases}

Diarrheal diseases cause over 40 percent of the deaths in disaster and refugee camp settings. Epidemics among victims are commonly related to polluted water sources (faecal contamination) or contamination of water during transportation and storage. Outbreaks have also been related to shared water containers and cooking pots, scarcity of soap and contaminated food, as well as preexisting poor sanitary infrastructures, water supply and sewerage systems. Floods are recurrent in many countries like Bangladesh and usually lead to a significant increase in diarrheal disease incidences. Following the 2005 earthquake in Pakistan, the Bam earthquake in 2003 in Iran, 2004 tsunami in Indonesia and 2001 earthquake in El Salvador showed outbreak of diarrheal infections. An evolving cholera epidemic was reported 9 months after the earthquake in Haiti with a high fatality rate.
Only a small cluster of Norovirus cases was reported in evacuation centers some weeks after the Great Eastern Japanese Earthquake and tsunami, while various pathogens were confirmed among the populations displaced by Hurricanes Allison (2001) and Katrina in the US.

\section{Diseases associated with crowding}

Measles: Crowded living conditions facilitate measles transmission. The risk for transmission after a natural disaster are dependent on baseline immunization coverage among the affected population and in particular among children $<15$ years of age and necessitate even higher immunization coverage levels to prevent outbreaks. A measles outbreak in the Philippines in 1991 among persons displaced by the eruption of $\mathrm{Mt}$. Pinatubo involved $>18,000$ cases. ${ }^{5}$ After the tsunami in Aceh, a cluster of measles involving 35 cases occurred in Aceh Utara district and continuing sporadic cases and clusters were common despite mass vaccination campaigns. ${ }^{5}$ In Pakistan, after the 2005 South Asia earthquake, sporadic cases and clusters of measles $(>400$ clinical cases in the 6 months after the earthquake) also occurred.

Neisseria meningitides meningitis is transmitted from person to person, particularly in situations of crowding. Cases and deaths from meningitis among those displaced in Aceh and Pakistan have been documented. Prompt response with antimicrobial prophylaxis, as occurred in Aceh and Pakistan, can interrupt transmission.

$\boldsymbol{A R I}$ Acute respiratory infections (ARI) are a major cause of illness and death among displaced populations, particularly in children $<5$ years of age. The reported incidence of ARI increased 4-fold in Nicaragua in the 30 days after Hurricane Mitch in 1998 and ARI accounted for the highest number of cases and deaths among those displaced by the tsunami in Aceh in 2004 and by the 2005 earthquake in Pakistan.

Other diseases associated with natural disasters

Tetanus Contaminated wounds, particularly in populations where vaccination coverage levels are low, are associated with illness and death from tetanus. A cluster of 106 cases of tetanus, including 20 deaths, occurred in Aceh and peaked 2-1/2 weeks after the tsunami . Cases were also reported in Pakistan following the 2005 earthquake. 
Fungal infections: An unusual outbreak of coccidiomycosis occurred after the January 1994 Southern California earthquake. It is caused by the fungus Coccidioidesimmitis, which is found in soil in certain semiarid areas of North and South America. This outbreak was associated with exposure to increased levels of airborne dust subsequent to landslides in the aftermath of the earthquake.

\section{Phases of outbreak and classification of infectious diseases}

Three clinical phases of natural disasters summarize the chronological public health effects on injured people and survivors:

- Phase (1), the impact phase (lasting up to to 4 days), is usually the period when victims are extricated and initial treatment of disaster-related injuries is provided.

- Phase (2), the post-impact phase (4 days to 4 weeks), is the period when the first waves of infectious diseases (air-borne, food-borne and/or water-borne infections) might emerge.

- Phase (3), the recovery phase (after 4 weeks), is the period when symptoms of victims who have contracted infections with long incubation periods or those with latent-type infections may become clinically apparent. During this period, infectious diseases that are already endemic in the area, as well as newly imported ones among the affected community, may grow into an epidemic.

\section{The long-term health conditions}

Typically addressed during the redevelopment phase include mental and psychological issues, vaccinating and eliminating the outbreak of communicable diseases like cholera, malaria and measles and reinstating the infrastructure of the health services system.

\section{Mental healthdisorders}

In the weeks and months following a disaster event, many patients will suffer from some psychological disorders such as post-traumatic stress disorder (PTSD) or general anxiety disorder (GAD). In many instances victims and survivors complain of some of the following symptoms: night terrors, sudden phobias, grief, depression, guilt, insomnia, loss of appetite or flashbacks and hallucinations. It is common for many behavioral responses - to disasters and catastrophic events - to remain subtly buried within a victim's coping mechanisms, surfacing only under close observation of key health related habits such as sleeping, eating, smoking or alcohol consumption.

Mental health problems rarely manifest themselves in the early stages of the emergency. But then you hope and expect to see people returning to some kind of normalcy; picking up and trying to dig out and get themselves into their houses and back to school or back to work. And when that doesn't happen then that's a sign the individual is beginning to experience posttraumatic stress disorder, maybe depression or may be anxiety.

It is for this reason health professionals urge people in natural disaster ridden communities to return to their daily routines as soon as possible.

\section{Communicable diseases}

Communities reeling from natural disasters also tend to become breeding grounds for outbreaks of communicable diseases, which are defined as diseases that easily transfer from person to person or animal to person. Continuing problems with hygiene and diseases related to hygiene are common in refugee camps.

\section{Waterborne disease}

a) Diarrheal disease outbreaks can occur after drinking water has been contaminated. An outbreak of diarrheal disease after flooding in Bangladesh in 2004 involved $>17,000$ cases; Vibrio cholerae (O1 Ogawa and O1 Inaba) and enterotoxigenic Escherichia coli were isolated. ${ }^{6}$ A large $(>16,000$ cases) cholera epidemic (O1 Ogawa) in West Bengal in 1998 was attributed to preceding floods and floods in Mozambique in January-March 2000 led to an increase in the incidence of diarrhea.

In Indonesia in 1992-1993, flooding was identified as a significant risk factor for diarrheal illnesses caused by Salmonella enteric serotype paratyphe A (paratyphoid fever). In a separate evaluation of risk factors for infection with Cryptosporidium parvum in Indonesia in 2001-2003, case-patients were $>4 \times$ more likely than controls to have been exposed to flooding. 
b) Hepatitis: In hepatitis E endemic areas, outbreaks frequently follow heavy rains and floods; the illness is generally mild and self-limited, but in pregnant women case-fatality rates can reach $25 \%$. After the 2005 earthquake in Pakistan over 1,200 cases of acute jaundice, many confirmed as hepatitis E, occurred among the displaced .Clusters of both hepatitis A and hepatitis E were noted in Aceh after the December 2004 tsunami.

c) Leptospirosis: Outbreaks of leptospirosis occurred in Taiwan, Republic of China associated with Typhoon Nali in 2001; in Mumbai, India, after flooding in 2000; in Argentina after flooding in 1998; and in the Krasnodar region of the Russian Federation in 1997.

\section{Vector borne diseases ${ }^{7}$}

Natural disasters, particularly meteorological events such as cyclones, hurricanes and flooding, landslides/ mudslides can affect vector-breeding sites and vector borne disease transmission. While initial flooding may wash away existing mosquito-breeding sites, standing water caused by heavy rainfall or overflow of rivers can create new breeding sites. This situation can result (with typically some weeks' delay) in an increase of the vector population and potential for disease transmission, depending on the local mosquito vector species and its preferred habitat. The crowding of infected and susceptible hosts, a weakened public health infrastructure and interruptions of ongoing control programs are all risk factors for vector borne disease transmission.

Malaria outbreaks in the wake of flooding are a wellknown phenomenon. An earthquake in Costa Rica's Atlantic Region in 1991 was associated with changes in habitat that were beneficial for breeding and preceded an extreme rise in malaria cases. Additionally, periodic flooding linked to El Niño-Southern Oscillation has been associated with malaria epidemics in the dry coastal region of northern Peru.

Dengue and chikungunya transmission is influenced by meteorological conditions, including rainfall and humidity and often exhibits strong seasonality. However, transmission is not directly associated with flooding. Such events may coincide with periods of high risk for transmission and may be exacerbated by increased availability of the vector's breeding sites (mostly artificial containers, fresh water logging in pits of road, building, trees) caused by disruption of basic water supply and solid waste disposal services. The risk for outbreaks can be influenced by other complicating factors, such as changes in human behavior (increased exposure to mosquitoes while sleeping outside, movement from dengue/chikungunya-non endemic to endemic areas, a pause in disease control activities, overcrowding) or changes in the habitat that promote mosquito breeding (landslide, deforestation, river damming and rerouting of water).

Although it is not possible to predict with accuracy which diseases will occur following certain types of disasters, diseases can be distinguished as either waterborne, air-borne/droplet or vector-borne diseases and contamination from wounded injuries.

\section{Disaster response: Mitigation/prevention of diseases} Effective treatment of mass casualties depends on local preparedness and requires triage of patients to treat those most likely to benefit first.

In disaster research and analysis, the vulnerability of a community is defined as the "characteristics of a person or group in terms of their capacity to anticipate, cope with, resist and recover from the impact of a natural hazard", a society must establish and adequately practice a "Health Disaster Management Program". One fourth of our populations are affected by cyclone and $80 \%$ live in flood prone regions.

Mortality and morbidity from these events have fallen substantially in the last 50 years, partially because of improvement of disaster management. Ministry of Disaster Management and Relief (MoDMR) of Bangladesh formulated the $7^{\text {th }}$ five year (2016-2021) plan of climate change and disaster management.

An effective disaster mitigation program should identify 1) endemic and epidemic diseases that are common in the affected area; 2) living conditions of the affected population, including number, size, location and density of settlements; 3 ) availability of safe water and adequate sanitation facilities; 4) underlying nutritional status and immunization coverage among the population; and 5) degree of access to healthcare and to effective case management.

\section{Diseases favoured by our climate}

Aedis egyptii mosquito spread three diseases; all three has been detected in Bangladesh. Dengue was first 
detected in Dhaka in 1999, Chikungunya in 2008 in Paba, Rajshahi and Zika in 2014 in Chittagong. Other disease with public health concern are viral infections like Bird flu (first detectd 2007), Swine flu 2009, ${ }^{8}$ Nipah, Japanese encephalitis and the bacterial infection melioidosis

Mellioidosis 9 : Burkholderia pseudomelli was isolated from Gazipur soil in July 2013, since then we have became $18^{\text {th }}$ definite country of Melioidosis. The disease was first described in 1941 in Myanmer. We have so far only 35 reported confirmed cases in our country. We have mentioned this disease in editorial of Vol no of our Journal (BIRDEM Medical Journal)

\section{Viral disease}

Nipah: This RNA virus, zoonotic disease was first detected in Bangladesh in 2001. The disease is transmitted by date palm sap contaminated by infected fruit bats. Human to human infection is possible. The sporadic cases has been detected in 2007 onwards. Nipah is associated with encephalitis. It presents with 3-14 days high fever and mental confusion which can progress to coma in days. Some may have respiratory symptoms in initial period. It is a disease with high mortality. Long term sequel with persistant epilepsy, personality disorder is common.

Japanese encephalitis: Caused by JEV-Japanese encephalitis virus. It is a disease of public health concern in 24 at risk countries in Asia. The disease burden has been reduced by imroved socio economic condition, immunisation and vaccination programme. It is transmitted by culex mosquito, man is the susceptible host affected by the disease. The virus can multiply in pig, cattle, birds (king stork) specially those animals move in mud and water. These are amplifying hosts only.

It causes fever and encephalitis. It can cause mental symptoms (cognitive disorder), permanent neurological sequels' like epilepsy and movement disoders. Majority of cases remain asymptomatic.

There is no WHO estimated prevalence of Japanese encephalitis in Bangladesh.

\section{Flu}

Bird flu/avian flu: This H5N1 viral infection was first detected in Bangladesh in 2007. Main sufferers are paultry firms workers. Death of chicken caused a havoc.
Human to human transmission is almost nil. Bird to human transmission is also rare, thus bird flu did not pause a threat to human being.

Swine flu: The 2009 pandemic H1N1 viral infection is a great threat to human being as human to human infection is possible. Its severe acute respiratory syndrome (SARS) variety is very dangerous which need good ICU support and is almost always fatal. Other categories like influenza like symptom (ILI) is like ordinary flu. The third one poses threat to persons with compromised immunity. Pregnant woman remain with great threat.

Both the flue are preventable with good personal hygiene. There is an antiviral agent oseltamavir with good therapeutic effect if started in earliest time (3-4 days) of onset of disease. There is special Flu vaccine also.

Dengue and Chikungunya were once considered to be same disease. These two diseases has become the public health concern in recent days. Both are related to the monsoon rain which favours the breeding of the mosquito. Prevalence dengue is stiil high though public awareness has been increased and the panic like its initial days is no longer prevailing. Both the disease present with fever. Mortality is the main concern with dengue as the patient may have fever, bleeding and may go into shock. Whereas morbidity is more with Chikungunya with high incidence of joint pain (arthritis /arhthralgia) with which patient can suffer from long time without any permanent disability/destruction of joints. Patient with dengue may have pain all over (not predominantly joints) and characteristically the retro orbital pain. Pain sometimes so intense that it was named as breaking bone disease. Chikungunya patient may bend up (stoop forward) with pain or may limp while walking.

Prevention is possible of these diseases. Vector (mosquito) control is here main aspect.

There is no vaccine for Chikungunya but the first dengue vaccine Dengavxia (CYD-TDV), a live recombinant tetravalent vaccine has been evaluated as a three dose series in phase III clinical trials and first registered in Mexico in December 2015. So far Dengavxia has been approved for use in 10 countries of Latin America and Asia with considerable achievement. Five other vaccines are in pipeline. 


\section{Conclusion}

Historically, fears of major disease outbreaks in the aftermath of natural disasters have shaped the perceptions of the public and policymakers. These expectations, misinformed by associations of disease with dead bodies, can create fear and panic in the affected population and lead to confusion in the media and elsewhere.

The risk for outbreaks after natural disasters is low, particularly when the disaster does not result in substantial population displacement. Five (5) priority measures that can reduce the risk for communicable disease transmission in populations affected by natural disasters are 1. Safe water, sanitation, site planning.2. Primary healthcare services 3. Surveillance/early warning system 4.Immunization 5. Prevention of malaria and Dengue and Chikungunya by controlling the vector Mosquitos. We have no control on nature only can take precautionary measure with dvolpoment of infrastructure and strong commitment. Changes of climate predisposes infectious diseases. Early apprehension, adequate and appropriate public and personal health measure mostly succeed in combating these tropical diseases.

\section{References}

1. Epidemics caused by dead bodies.de Ville de Goyet C: a disaster myth that does not want to die. Rev Panam Salud Publica 2004;15:297-99

2. Medical and Public Health Consequences of Natural and Biological Disasters. Eric K. Noji.Natural Hazards Review2003;2(3): 143-56.

3. Center for Disease Control Website - National Center for Environmental Health - Hurricane - A prevention guide to promote your personal health and safety http://md.skolar.com/ index_.jsp?trg $=\% 2$ Fshine $\% 2$ Fhome $\% 2$ Fsearch.jsp\&nav=home

4. Increased acute myocardial infarction mortality following the 1995 Great Hanshin-Awaji earthquake in Japan.Ogawa K, Tsuji I, Shono K, Hisamichi S., Int J Epidemiol 2000 ; 29 (3): 449-55.

5. Measles transmission and vaccine effectiveness during a large outbreak on a densely populated island: implications for vaccination policy. Marin M, Nguyen HQ, Langidrik JR, Edwards R, Briand K, Papania MJ, et al.. Clin Infect Dis 2006; 42:315-19 .

6. EnterotoxigenicEscherichia coli and Vibrio cholerae diarrhea, Bangladesh. Qadri F, Khan AI, Faruque ASG, Begum YA, Chowdhury F, Nair GB, et al. Emerg Infect Dis 2005;11: 1104-1107.

7. Lifson AR. Mosquitoes, models, and dengue. Lancet 1996; 347:1201-1202

8. Deshe shathti nutan rog. Prothom alo,daily bangla news paper 2017; July $21: 2$.

9. Melioidosis (editorial). BIRDEM Med J 2016;6(2). 